\title{
Finslerian Ricci Deformation and Conformal Metrics
}

\author{
Gilbert Nibaruta $^{1 *}$, Serge Degla ${ }^{2}$, Léonard Todjihounde ${ }^{1}$ \\ ${ }^{1}$ Institut de Mathématiques et de Sciences Physiques, Porto-Novo, Bénin \\ ${ }^{2}$ Ecole Normale Supérieure de Natitingou, Natitingou, Bénin \\ Email: ^gilbert.nibaruta@imsp-uac.org, deglaserge@yahoo.fr, leonardt@imsp-uac.org
}

How to cite this paper: Nibaruta, G., Degla, S. and Todjihounde, L. (2018) Finslerian Ricci Deformation and Conformal Metrics. Journal of Applied Mathematics and Physics, 6, 1522-1536. https://doi.org/10.4236/jamp.2018.67128

Received: January 28, 2018

Accepted: May 5, 2018

Published: July 27, 2018

Copyright (c) 2018 by authors and Scientific Research Publishing Inc. This work is licensed under the Creative Commons Attribution International License (CC BY 4.0).

http://creativecommons.org/licenses/by/4.0/

\begin{abstract}
In this paper, a new Ricci flow is canonically introduced in Finsler Geometry and, under the variance of Finsler-Ehresmann form, conformal changes of Finsler metrics are studied. Some existence conditions of this Finslerian Ricci flow on a compact manifold which preserves the conformal class of the initial metric are obtained as an application.
\end{abstract}

\section{Keywords}

Ehresmann Connection, Ricci Flow, Trace-Free Ricci Tensor, Conformal Change of Finsler-Ehresmann Form

\section{Introduction}

The Ricci flow is a very powerful tool in studying of the geometry of manifolds and has many applications in Mathematics and Physics. In Finsler Geometry, the problems on Ricci flow are very interesting. In 2017, to study deformation of Cartan curvature, Bidabad, Shahi and Ahmadi considered the Akbar-Zadeh's Ricci curvature and introduced certain Ricci flow for Finsler $n$-manifolds [1].

In this paper, we use the pulled-back bundle approach [2] to introduce a Finslerian horizontal Ricci flow, called Finslerian Ricci deformation. This approach is natural and is very important. The problem of construction of the Finslerian Ricci deformation contains a number of new conceptual. Let $(M, F)$ be a Finsler manifold of flag scalar curvature. Then we consider a Finslerian Ricci tensor defined by $\mathbf{R i c}_{F}^{H}(\xi, X):=\sum_{a=1}^{n} \mathbf{R}\left(\xi, e_{a}, X, \hat{e}_{a}\right)$. In this definition, $\mathbf{R i c}_{F}^{H}$ denotes again a Ricci tensor associated with $F$ and a Finsler-Ehresmann connection $\mathcal{H}, \xi$ is a section of the vector bundle $\pi^{*} T M, X$ is a section of the tangent bundle $T \stackrel{T}{T} M$ of $\stackrel{\circ}{T} M:=T M \backslash\{0\}, \mathbf{R}$ is the $h h$-curvature of Chern 
connection, $\left\{e_{a}\right\}_{a=1, \cdots, n}$ is the special $g$-orthonormal basis section for $\pi^{*} T M$ and $\left\{\hat{e}_{a}\right\}_{a=1, \cdots, n}$ is the orthonormal basis section for $\mathcal{H}$. Let $\left(M, F_{t}\right)$ be an $n$-dimensional Finsler manifold and $\left\{g_{t}\right\}_{t \in[0, \tau)}$ ( $\tau$ being a finite parameter $)$ a family of fundamental tensors of $\left\{F_{t}\right\}_{t \in[0, \tau)}$. We consider the following Finslerian Ricci deformation:

$$
\frac{\partial}{\partial t} g_{t}=-2 \mathbf{R i c}_{F_{t}}^{H},\left.\quad g_{t}\right|_{0}=g, \quad \forall t \in[0, \tau) .
$$

The existence and uniqueness for solution of the Equation (1) are known in special cases, particulary in Riemannian spaces and Berwald spaces [3]. This Finslerian Ricci deformation generalizes the classical Riemannian one.

A Finsler metric $\tilde{F}$ on $M$ is said to be conformally equivalent to $F$, if there exists a $C^{\infty}$ function $u$ on $M$ such that $\tilde{F}=e^{u} F$. In this paper, $[F]$ denotes the conformal class of $F$. We prove the following results.

Theorem 1. Let $(M, F)$ be an n-dimensional compact Finsler manifold, $F_{t}$ a Ricci deformation of $F$ for $t \in[0, \tau)$ and $g_{t}$ the fundamental tensor of $F_{t}$. If $\left[F_{t}\right]=[F]$ for all $t \in[0, \tau)$ then there exists a family $\left\{u_{t}\right\}_{t \in[0, \tau)}$ of $C^{\infty}$ functions on $\mathrm{M}$ satisfying the following equation.

$$
\begin{aligned}
\frac{\partial u_{t}}{\partial t}= & -\frac{1}{n} e^{-2 u_{t}}\left[\operatorname{Scal}_{F}^{H}+2(n-1) \Delta^{H} u_{t}-(n-1)(n-2)\left\|\nabla u_{t}\right\|_{g}^{2}\right] \\
& -\frac{2-n}{n} e^{-2 u_{t}} \sum_{a=1}^{n}\left[g\left(\nabla u_{t}, \Theta\left(\hat{e}_{a}, \hat{e}_{b}\right)\right)-g\left(\Theta\left(\hat{e}_{a}, \mathbf{h}\left(\nabla u_{t}\right)\right), e_{a}\right)\right] \\
& -\frac{1}{n} e^{-2 u_{t}} \sum_{a, b=1}^{n}\left[g\left(\Theta\left(\hat{e}_{b}, \mathbf{h}\left(\Theta\left(\hat{e}_{a}, \hat{e}_{b}\right)\right)\right), e_{a}\right)-g\left(\Theta\left(\hat{e}_{a}, \mathbf{h}\left(\Theta\left(\hat{e}_{a}, \hat{e}_{b}\right)\right)\right), e_{a}\right)\right] \\
& -\frac{1}{n} e^{-2 u_{t}} \sum_{a, b=1}^{n}\left[g\left(\left(\nabla_{\hat{e}_{b}} \Theta\right)\left(\hat{e}_{a}, \hat{e}_{b}\right), e_{a}\right)-g\left(\left(\nabla_{\hat{e}_{a}} \Theta\right)\left(\hat{e}_{b}, \hat{e}_{b}\right), e_{a}\right)\right]
\end{aligned}
$$

with $\left.u_{t}\right|_{t=0}=0$, where $\mathbf{S c a l}_{F}^{H}$ is the horizontal scalar curvature, $\nabla$ is the Chern connection, $\mathbf{h}$ denotes a horizontal lift of a section of $\pi^{*} T M, \Delta^{H}$ is the horizontal Laplacian, $\nabla$ is the gradient and $\Theta$ is the $(0,2 ; 1)$-tensor on $M$ measuring the variation of $\nabla$ during the conformal change of $F$.

Corollary 1. Assume $n \geq 2$. Then the Equation (2) in Theorem 1 has a unique solution on a parameterized-interval $[0, s)$ for some $s \leq \tau$.

Finally, using the trace-free horizontal Ricci tensor we prove the following

Theorem 2. Let $(M, F)$ be an $n$-dimensional compact Finsler-Einstein manifold, $g$ its fundamental tensor and $u_{t}$ the unique solution of Equation (2) on $[0, s) \times M$ for some $s \leq \tau$, where $[0, \tau)$ is the maximal parameterized-interval on which (1) has a solution. Assume that the tensor $T_{t}$, defined for a $t \in[0, s)$ and in local coordinate by

$$
\begin{aligned}
T_{c d}(t)= & e^{-2 u_{t}}\left\{-(n-2)\left[H_{u_{t}}\left(e_{c}, e_{d}^{H}\right)-d u_{t}\left(e_{c}\right) d u_{t}\left(e_{d}\right)\right]\right. \\
& +\sum_{a=1}^{n}\left[(2-n) g\left(\nabla u_{t}, \Theta\left(\hat{e}_{a}, \hat{e}_{a}\right)\right)-g\left(\Theta\left(\mathbf{h}\left(\nabla u_{t}\right), \hat{e}_{a}\right), e_{a}\right)\right] \delta_{c d} \\
& +\sum_{a=1}^{n}\left[-g\left(\Theta\left(\hat{e}_{a}, \mathbf{h}\left(\nabla u_{t}\right)\right), e_{a}\right)+g\left(\Theta\left(\hat{e}_{a}, \mathbf{h}\left(\nabla u_{t}\right)\right), e_{a}\right)\right] \delta_{c d}
\end{aligned}
$$




$$
\begin{aligned}
& +\sum_{a, b=1}^{n}\left[g\left(\Theta\left(\hat{e}_{b}, \mathbf{h}\left(\Theta\left(\hat{e}_{a}, \hat{e}_{b}\right)\right)\right), e_{a}\right)-g\left(\Theta\left(\hat{e}_{a}, \mathbf{h}\left(\Theta\left(\hat{e}_{a}, \hat{e}_{b}\right)\right)\right), e_{a}\right)\right] \delta_{c d} \\
& \left.+\sum_{a, b=1}^{n}\left[g\left(\left(\nabla_{\hat{e}_{b}} \Theta\right)\left(\hat{e}_{a}, \hat{e}_{b}\right), e_{a}\right)-g\left(\left(\nabla_{\hat{e}_{a}} \Theta\right)\left(\hat{e}_{b}, \hat{e}_{b}\right), e_{a}\right)\right] \delta_{c d}\right\},
\end{aligned}
$$

is conformally equivalent to $g$. Then there exists a unique Ricci deformation $\left\{F_{t}\right\}_{t \in[0, s)}$ of $F$ such that $\left[F_{t}\right]=[F]$.

The rest of this paper is organised as follows. In Section 2, we give some basic notions on Finsler manifolds. In Section 3, we prove the main results given above.

\section{Preliminaries}

In order to deal with the Finslerian Ricci deformation, it is preferable to use a global definition of Chern connection. We adopt the notations given in [2] and $[4]$.

Let $\pi: T M \rightarrow M$ be the tangent bundle of a connected $C^{\infty}$ manifold of dimension $n \geq 3$. We denote by $\stackrel{\circ}{T} M=T M \backslash\{0\}$ the slit tangent bundle of $M$. We introduce a coordinate system on $T M$ as follows. Let $U \subset M$ be an open set with $\left(x^{i}\right)_{i=1, \cdots, n}$ a local coordinate on $U$. By setting $y=y^{i} \frac{\partial}{\partial x^{i}}$ for every $y \in \pi^{-1}(U)$, we introduce a local coordinate $\left(x^{i}, y^{i}\right)_{i=1, \cdots, n}$ on $\pi^{-1}(U) \subset T M$. Throughout this paper, we use Einstein summation convention for the expressions with indices when an index appears twice as a subscript as well as a superscript in a term.

Definition 2.1. A function $F: T M \rightarrow[0, \infty)$ is called a Finsler metric on $M$ if:

1) $F$ is $C^{\infty}$ on the entire slit tangent bundle $\stackrel{\circ}{T} M$,

2) $F$ is positively 1-homogeneous on the fibers of $T M$, that is $\forall c>0, F(x, c y)=c F(x, y)$,

3) the Hessian matrix $\left(g_{i j}(x, y)\right)_{1 \leq i, j \leq n}$ with elements

$$
g_{i j}(x, y):=\frac{1}{2} \frac{\partial^{2} F^{2}(x, y)}{\partial y^{i} \partial y^{j}}
$$

is positive definite at every point $(x, y)$ of $\stackrel{\circ}{T} M$.

Given a manifold $M$ and a Finsler metric $F$ on $T M$, the pair $(M, F)$ is called a Finsler manifold.

Remark 2.1. $F(x, y) \neq 0$ for all $x \in M$ and for every $y \in T_{x} M \backslash\{0\}$.

The pulled-back bundle $\pi^{*} T M$ is a vector bundle over the slit tangent bundle $\stackrel{\circ}{T} M$. The fiber at a point $(x, y) \in \stackrel{\circ}{T} M$ is defined by

$$
\left.\pi^{*} T M\right|_{(x, y)}:=\left.\left\{(x, y, v) \mid v \in T_{\pi(x, y)} M\right\}\right|_{(x, y)} \cong T_{x} M .
$$

By the objects (4), the vector bundle $\pi^{*} T M$ admits a Riemannian metric. $g:=g_{i j} d x^{i} \otimes d x^{j} \quad$ called fundamental tensor. Likewise, there is the Finslerian Cartan tensor 


$$
\mathcal{A}=\mathcal{A}_{i j k} d x^{i} \otimes d x^{j} \otimes d x^{k}
$$

where $\mathcal{A}_{i j k}:=\frac{F}{2} \frac{\partial g_{i j}}{\partial y^{k}} . \mathcal{A}$ is a symmetric section of $\otimes^{3} \pi^{*} T^{*} M$. Note that, $\frac{\partial}{\partial x^{i}}$ and $d x^{i}$ are respectively regarded as basis sections of $\pi^{*} T M$ and $\pi^{*} T^{*} M$ (see [2]).

\subsection{Finsler-Ehresmann Connection and Chern Connection}

For the differential mapping $\pi_{*}$ of the submersion $\pi: \stackrel{\circ}{T} M \rightarrow M$, the vertical subbundle $\mathcal{V}$ of $T \stackrel{\circ}{T M}$ is defined by $\mathcal{V}:=\operatorname{ker}\left(\pi_{*}\right)$ and it is locally spanned by the set $\left\{F \frac{\partial}{\partial y^{i}}, 1 \leq i \leq n\right\}$, on each $\pi^{-1}(U) \subset T M$. Then it induces the short exact sequence

$$
0 \rightarrow \mathcal{V} \stackrel{i}{\longrightarrow} T \stackrel{\circ}{T} M \stackrel{\pi_{*}}{\longrightarrow} \pi^{*} T M \rightarrow 0
$$

A horizontal subspace $\mathcal{H}$ of $T \stackrel{0}{T} M$ is by definition any complementary to $\mathcal{V}$. The bundles $\mathcal{H}$ and $\mathcal{V}$ give a smooth splitting [5]

$$
T \stackrel{\circ}{T} M=\mathcal{H} \oplus \mathcal{V} \text {. }
$$

The vertical bundle $\mathcal{V}$ is uniquely determined but the horizontal bundle $\mathcal{H}$ is not canonically determined. An Ehresmann connection is a selection of horizontal subspace $\mathcal{H}$ of $T \stackrel{\circ}{T} M$.

In this paper, we consider the choice of Ehresmann connection which arises from the Finsler metric $F$ and it is call Finsler-Ehresmann connection [6], constructed as follows. As explained in [7], all Finsler structure $F$ on $M$ induces a vector field on $\stackrel{\circ}{T} M$ in the form

$$
G(x, y)=y^{i} \frac{\partial}{\partial x^{i}}-2 G^{i}(x, y) \frac{\partial}{\partial y^{i}}
$$

where $x=\left(x^{i}\right) \in M, y=y^{i} \frac{\partial}{\partial x^{i}} \in \stackrel{\circ}{T} M$ and the elements

$$
G^{i}(x, y):=\frac{1}{4} g^{i l}\left(\frac{\partial g_{j l}}{\partial x^{k}}+\frac{\partial g_{l k}}{\partial x^{j}}-\frac{\partial g_{j k}}{\partial x^{l}}\right) y^{j} y^{k}
$$

are $y$-homogeneous of degree two. The vector field $G$ is called spray on $M$ and the $G^{i}, i=1, \cdots, n$ are called spray coefficients of $G$.

Consider the functions

$$
N_{j}^{i}(x, y):=\frac{\partial G^{i}}{\partial y^{j}}(x, y), 1 \leq i, j \leq n
$$

one defines a $\pi^{*} T M$-valued $C^{\infty}$ form on $\stackrel{\circ}{T} M$ by

$$
\theta=\frac{\partial}{\partial x^{i}} \otimes \frac{1}{F}\left(d y^{i}+N_{j}^{i} d x^{j}\right) .
$$

This $\pi^{*} T M$-valued $C^{\infty}$ form $\theta$ is globally well defined on $\stackrel{\circ}{T} M$ [4].

From the form $\theta$, called Finsler-Ehresmann form, defined in (9), one defines 
a Finsler-Ehresmann connection as follows.

Definition 2.2. A Finsler-Ehresmann connection associated with the submersion.

$\pi: \stackrel{\circ}{T} M \rightarrow M$ is the subbundle $\mathcal{H}$ of $T \stackrel{\circ}{T} M$ given by $\mathcal{H}:=\operatorname{ker} \theta$, where $\theta$ is the bundle morphism from $T \stackrel{\circ}{T} M$ to $\pi^{*} T M$ defined in (9), and which is complementary to the vertical subbundle $\mathcal{V} \subset T \stackrel{\circ}{T} M$.

Now we define horizontal lift and vertical lift of a section of $\pi^{*} T M$ as follows.

Definition 2.3. Let $\pi: \stackrel{\circ}{T} M \rightarrow M$ be the restricted projection.

1) The form $\theta: T \stackrel{T}{T M} \rightarrow \pi^{*} T M$ induces a linear map

$$
\left.\theta\right|_{(x, y)}: T_{(x, y)} \stackrel{\circ}{T} M \rightarrow T_{x} M, \text { for each point }(x, y) \in \stackrel{\circ}{T} M
$$

where $x=\pi(x, y)$.

The vertical lift of a section $\xi$ of $\pi^{*} T M$ is a unique section $v(\xi)$ of $T \stackrel{\circ}{T M}$ such that for every $(x, y) \in \stackrel{\circ}{T} M$

$$
\left.\pi_{*}(\boldsymbol{v}(\xi))\right|_{(x, y)}=0_{(x, y)} \text { and } \theta(\boldsymbol{v}(\xi))_{(x, y)}=\xi_{(x, y)} .
$$

2) The differential projection $\pi_{*}: T \stackrel{\circ}{T} M \rightarrow \pi^{*} T M$ induces a linear map

$$
\left.\pi_{*}\right|_{(x, y)}: T_{(x, y)} \stackrel{\circ}{T} M \rightarrow T_{x} M, \text { for each point }(x, y) \in \stackrel{\circ}{T} M ;
$$

where $x=\pi(x, y)$.

The horizontal lift of a section $\xi$ of $\pi^{*} T M$ is a unique section $\boldsymbol{h}(\xi)$ of $T \stackrel{\circ}{T M}$ such that for every $(x, y) \in \stackrel{\circ}{T} M$

$$
\left.\pi_{*}(\boldsymbol{h}(\xi))\right|_{(x, y)}=\xi_{(x, y)} \text { and }\left.\pi_{*}(\boldsymbol{h}(\xi))\right|_{(x, y)}=\xi_{(x, y)} .
$$

Remark 2.2. The vector bundle $\pi^{*} T M$ can be naturally identified with the horizontal subbundle $\mathcal{H}$ of $T \stackrel{\circ}{T} M$ or with the vertical bundle $\mathcal{V}$. Thus any section $\xi$ of $\pi^{*} T M$ is considered as a section of $\mathcal{H}$ or a section of $\mathcal{V}$. In fact

$$
\xi=\xi^{i} \frac{\partial}{\partial x^{i}} \in \Gamma\left(\pi^{*} T M\right) \Leftrightarrow \boldsymbol{h}(\xi)=\xi^{i} \frac{\delta}{\delta x^{i}} \in \Gamma(\mathcal{H})
$$

and

$$
\xi=\xi^{i} \frac{\partial}{\partial x^{i}} \in \Gamma\left(\pi^{*} T M\right) \Leftrightarrow \boldsymbol{v}(\xi)=\xi^{i} F \frac{\partial}{\partial y^{i}} \in \Gamma(\mathcal{V})
$$

where

$$
\left\{\frac{\delta}{\delta x^{i}}:=\frac{\partial}{\partial x^{i}}-N_{i}^{j} \frac{\partial}{\partial y^{j}}=h\left(\frac{\partial}{\partial x^{i}}\right)\right\}_{i=1, \cdots, n} \text { and }\left\{F \frac{\partial}{\partial y^{i}}:=v\left(\frac{\partial}{\partial x^{i}}\right)\right\}_{i=1, \cdots, n}
$$

are respectively horizontal and vertical lifts of the natural basis $\left\{\frac{\partial}{\partial x^{i}}\right\}_{i=1, \cdots, n}$ for $\pi^{*} T M$.

The following theorem defines the Chern connection on the bundle $\pi^{*} T M$.

Theorem 3. [4] Let $(M, F)$ be a Finsler manifold, $g$ the fundamental tensor associated with $F$ and $\pi_{*}$ the differential mapping of the submersion 
$\pi: \stackrel{\circ}{T} M \rightarrow M$. There exist a unique linear connection $\nabla$ on the pulled-back tangent bundle $\pi^{*} T M$ such that, for all

$$
X, Y \in \Gamma(T \stackrel{\circ}{T} M) \text { and } \xi, \eta \in \Gamma\left(\pi^{*} T M\right),
$$

one has the following properties:

1) Symmetry: $\nabla_{X} \pi_{*} Y-\nabla_{Y} \pi_{*} X=\pi_{*}[X, Y]$,

2) Almost $g$-compatibility:

$$
X(g(\xi, \eta))=g\left(\nabla_{X} \xi, \eta\right)+g\left(\xi, \nabla_{X} \eta\right)+2 \mathcal{A}(\theta(X), \xi, \eta),
$$

where $\mathcal{A}$ is the Cartan tensor defined in (6) and $\theta$ is the Finsler-Ehresmann form defined in (9).

\subsection{On Finslerian Curvatures of Chern Connection}

Let $\mathcal{E}$ be a vector bundle over $\stackrel{\circ}{T} M$. Then, one denotes by $\Gamma^{p}(\mathcal{E})$ the $C^{\infty}(T M)$-module of differentiable sections of $\mathcal{E}^{p}$, where

$$
\mathcal{E}^{p}=\underbrace{\mathcal{E} \oplus \cdots \mathcal{E}}_{p \text {-times }}
$$

denotes the fibered product of $\mathcal{E}$. By convention $\Gamma^{0}(\mathcal{E})=C^{\infty}(T M)$. The tensors that will be considered are defined as follows:

Definition 2.4. Let $(M, F)$ be a Finsler manifold. A tensor field $T$ of type $\left(p_{1}, p_{2} ; q\right)$ on $(M, F)$ is a mapping

$$
T: \underbrace{\pi^{*} T M \oplus \cdots \oplus \pi^{*} T M}_{p_{1} \text { times }} \oplus \underbrace{\stackrel{\circ}{T} M \oplus \cdots \oplus T \stackrel{\circ}{T} M}_{p_{2} \text {-times }} \rightarrow \otimes^{q} \pi^{*} T M, p_{1}, p_{2} \text { and } q \in \mathbb{N}
$$

which is $C^{\infty}(T M)$-linear in each arguments.

The full curvature $\phi$, of Chern connection $\nabla$, is the $(1,2 ; 1)$-tensor defined by

$$
\phi(X, Y) \xi=\nabla_{X} \nabla_{Y} \xi-\nabla_{Y} \nabla_{X} \xi-\nabla_{[X, Y]} \xi
$$

where $X, Y \in \Gamma(T \stackrel{\circ}{T} M)$ and $\xi \in \Gamma\left(\pi^{*} T M\right)$. Using the decomposition (8), we have

$$
\nabla_{X}=\nabla_{X^{H}}+\nabla_{X^{V}},
$$

where $X=X^{H}+X^{V}$ with $X^{H} \in \Gamma(\mathcal{H})$ and $X^{V} \in \Gamma(\mathcal{V})$.

The full curvature $\phi$ can be written as

$$
\phi(X, Y) \xi=\phi^{H H}(X, Y) \xi+\phi^{H V}(X, Y) \xi+\phi^{V H}(X, Y) \xi+\phi^{V V}(X, Y) \xi
$$

where

$$
\begin{aligned}
& \phi^{H H}(X, Y) \xi=\phi\left(X^{H}, Y^{H}\right) \xi=\nabla_{X^{H}} \nabla_{Y^{H}} \xi-\nabla_{Y^{H}} \nabla_{X^{H}} \xi-\nabla_{[X, Y]^{H}} \xi \\
& \phi^{H V}(X, Y) \xi=\phi\left(X^{H}, Y^{V}\right) \xi=\nabla_{X^{H}} \nabla_{Y^{V}} \xi-\nabla_{Y^{V}} \nabla_{X^{H}} \xi-\nabla_{\left[X^{H}, Y^{V}\right.} \xi \\
& \phi^{V H}(X, Y) \xi=\phi\left(X^{V}, Y^{H}\right) \xi=\nabla_{X^{V}} \nabla_{Y^{H}} \xi-\nabla_{Y^{H}} \nabla_{X^{V}} \xi-\nabla_{\left[X^{V}, Y^{H}\right.} \xi
\end{aligned}
$$




$$
\phi^{V V}(X, Y) \xi=\phi\left(X^{V}, Y^{V}\right) \xi=\nabla_{X^{V}} \nabla_{Y^{V}} \xi-\nabla_{Y^{V}} \nabla_{X^{V}} \xi-\nabla_{\left[X^{V}, Y^{V}\right.} \xi
$$

\section{Remark 2.3.}

1) As in the Riemannian case, one can define a $(2,2 ; 0)$ version of $\phi$ as follows:

$$
\begin{aligned}
\Phi(\xi, \eta, X, Y) & =g(\phi(X, Y) \xi, \eta) \\
& =g(R(X, Y) \xi, \eta)+g(P(X, Y) \xi, \eta) \\
& =\boldsymbol{R}(\xi, \eta, X, Y)+\boldsymbol{P}(\xi, \eta, X, Y)
\end{aligned}
$$

where $\boldsymbol{R}$ and $\boldsymbol{P}$ are respectively the $h h$ - and $h v$-curvature tensor of the Chern connection. One has

$$
\boldsymbol{R}(\xi, \eta, X, Y)=\Phi\left(\xi, \eta, X^{H}, Y^{H}\right)
$$

and

$$
\boldsymbol{P}(\xi, \eta, X, Y)=\Phi\left(\xi, \eta, X^{H}, Y^{V}\right)+\Phi\left(\xi, \eta, X^{V}, Y^{H}\right) .
$$

2) The $h h$-curvature tensor $\boldsymbol{R}$ is a generalization of the usual Riemannian curvature.

3) The $h v$-curvature tensor $\boldsymbol{P}$ is a Finsler non Riemannian curvature.

Definition 2.5. Let $(M, F)$ be a Finsler manifold, $R$ the horizontal part of the full curvature tensor associated with the Chern connection. We define

1) the horizontal Ricci tensor $\boldsymbol{R i c}_{F}^{H}$ of $(M, F)$ by

$$
\begin{aligned}
\boldsymbol{R i c}_{F}^{H}: & \pi^{*} T M \oplus T \stackrel{\circ}{T} M \rightarrow C^{\infty}(T M) \\
(\xi, X) & \mapsto \text { trace }_{g}(\eta \mapsto R(X, \boldsymbol{h}(\eta)) \xi),
\end{aligned}
$$

for every $\eta \in \Gamma\left(\pi^{*} T M\right)$. In $g$-orthonormal basis sections $\left\{e_{a}\right\}_{a=1, \cdots, n}$ of $\pi^{*} T M$, we have

$$
\boldsymbol{R i c}_{F}^{H}(\xi, X):=\sum_{a=1}^{n} \boldsymbol{R}\left(\xi, e_{a}, X, \hat{e}_{a}\right)
$$

2) the horizontal scalar curvature $\boldsymbol{S c a l}_{F}^{H}$ of $(M, F)$ is the trace of the horizontal Ricci tensor. $\boldsymbol{S c a l}_{F}^{H}$ is a function on $\stackrel{\circ}{T} M$. In $g$-orthonormal basis sections $\left\{e_{a}\right\}_{a=1, \cdots, n}$ of $\pi^{*} T M$,

$$
\boldsymbol{S c a l}_{F}^{H}:=\sum_{a=1}^{n} \boldsymbol{R i c}_{F}^{H}\left(e_{a}, \hat{e}_{a}\right)=\sum_{a, b=1}^{n} \boldsymbol{R}\left(e_{a}, e_{b}, \hat{e}_{a}, \hat{e}_{b}\right) .
$$

3) Let $(M, F)$ be a Finsler manifold and $g$ its fundamental tensor. Consider a flag $\mathcal{P} \subset T_{x} M$, that is a 2-dimensional subspace of $T_{x} M$, a flag-pole $0 \neq y \in T_{x} M$ and a transverse edge $\xi$. A flag curvature is defined by

$$
\boldsymbol{K}(x, y, \mathcal{P})=\boldsymbol{K}(x, y, \xi)=\frac{\boldsymbol{R}(y, \xi, X, Y)}{g(y, y) g(\xi, \xi)-(g(y, \xi))^{2}}
$$

where $\xi \in \mathcal{P}$ is a noncolinear to the vector $y$, with $X$ and $Y$ such that $\xi=\pi_{*} X$ and $y=\pi_{*} Y$.

Remark 2.4. If $K$ is independent of the transverse edge $\xi$, then the Finsler 
manifold $(M, F)$ is called of scalar flag curvature. Denote this scalar by $\tau=\tau(x, y)$. When $\tau(x, y)$ has no dependence on either $x$ nor $y,(M, F)$ is said to be of constant scalar curvature.

Now, we define the trace-free horizontal Ricci tensor and an Finsler-Einstein metric as follows.

Definition 2.6.

1) The trace-free horizontal Ricci tensor of an $n$-dimensional Finsler manifold $(M, F)$ is a $(1,1 ; 0)$-tensor on $(M, F)$ given by

$$
\boldsymbol{B}_{F}^{H}=\boldsymbol{R i c}_{F}^{H}-\frac{1}{n} \boldsymbol{S c a l}_{F}^{H} \underline{g},
$$

where $\underline{g}:=\pi^{*} g$ that is the pullback of $g$ by the submersion $\pi: \stackrel{\circ}{T} M \rightarrow M$; and for every $\xi \in \Gamma\left(\pi^{*} T M\right)$ and for any $X \in \Gamma(T \stackrel{\circ}{T} M), \underline{g}(\xi, X)=g\left(\xi, \pi_{*} X\right)$, $\boldsymbol{R i c}_{F}^{H}$ is the horizontal Ricci tensor and $\boldsymbol{S c a l}_{F}^{H}$ is the horizontal scalar curvature of $(M, F)$.

2) An $n$-dimensional Finsler manifold $(M, F)$ is horizontally Einstein if the trace-free horizontal Ricci tensor associated with $F$ vanishes, that is

$$
\boldsymbol{R i c}_{F}^{H}=\frac{1}{n} \boldsymbol{S c a l}_{F}^{H} \underline{g} .
$$

In this case, $\boldsymbol{S c a l}_{F}^{H}$ is a function on $M$ for $n \geq 3$.

\subsection{Horizontal Differential Operators on a Finsler Manifold}

In this paragraph, we give fundamental horizontal differential operators on $(M, F)$.

Remark 2.5. A differential operator $O$ of order $2 \mathrm{~m}$ defined on a differentiable manifold $M$ is written as

$$
O(u)=f\left(x, u, \nabla u, \nabla^{2 m} u\right)
$$

where $u \in C^{2 m}(M)$ and $f$ is assumed to be a differentiable function of its arguments.

\section{Definition 2.7.}

1) Let $\tau: \pi^{*} T M \rightarrow T M$ be the canonical mapping defined by $\tau(x, y, v)=v$. For a smooth function $u$ on $M$, the gradient of $u$, noted by $\nabla u$, is the section of $\pi^{*} T M$, given by

$$
g_{(x, y)}\left(\nabla u_{(x, y)}, \xi_{(x, y)}\right)=d u_{\pi(x, y)}(\tau \xi)
$$

for any $\xi \in \Gamma\left(\pi^{*} T M\right)$ and for every $(x, y) \in \stackrel{\circ}{T} M$. Locally, one has

$$
\nabla u_{(x, y)}=g^{i j}(x, y) \frac{\partial u}{\partial x^{i}} \frac{\partial}{\partial x^{j}} .
$$

2) For a $C^{\infty}$ section $\xi \in \Gamma\left(\pi^{*} T M\right)$, we define the horizontal divergence by

$$
\operatorname{div}^{H} \xi=\operatorname{trace}_{g}\left(\eta \mapsto \nabla_{h(\eta)} \xi\right),
$$

where $g$ is the fundamental tensor associated with $F$ and $\nabla$ is the Chern 
connection.

Remark 2.6. In the local basis sections $\left\{\frac{\partial}{\partial x^{i}}\right\}_{i=1, \cdots, n}$ of the bundle $\pi^{*} T M$, we have:

$$
\operatorname{div}^{H} \xi=g^{i j} g\left(\nabla_{\frac{\delta}{\delta x^{i}}} \xi, \frac{\partial}{\partial x^{j}}\right), \frac{\delta}{\delta x^{i}}:=\boldsymbol{h}\left(\frac{\partial}{\partial x^{i}}\right) .
$$

\section{Definition 2.8 .}

1) Let $(M, F)$ be a $C^{\infty}$ Finsler manifold and $\nabla$ the Chern connection on the pulled-back bundle $\pi^{*} T M$. The horizontal Hessian of a $C^{\infty}$ function $u$ on $M$ is the map

$$
H_{u}: \Gamma\left(\pi^{*} T M\right) \times \Gamma(T \stackrel{\circ}{T M}) \rightarrow C^{\infty}(T M)
$$

such that

$$
H_{u}(\xi, X)=g\left(\xi, \nabla_{X}(\nabla u)\right), \forall \xi \in \pi^{*} T M, \forall X \in T \stackrel{\circ}{T} M .
$$

2) Let $(M, F)$ be a $C^{\infty}$ Finsler manifold and $u$ a $C^{\infty}$ function on $M$. The horizontal Laplacian of $u$ are respectively defined by the following relation.

$$
\Delta^{H} u=-d i v^{H} \nabla u .
$$

Lemma 4. Let $u \in C^{\infty}(M)$. The horizontal Laplacian $\Delta^{H} u$ of $\mathrm{u}$ can be given in term of the horizontal Hessian of $\mathrm{u}$ by

$$
\Delta^{H} u=- \text { trace }_{g}\left((\xi, \eta) \mapsto H_{u}(\xi, \mathbf{h}(\eta))\right), \quad \xi, \eta \in \Gamma\left(\pi^{*} T M\right) .
$$

Furthermore, in $g$-orthonormale basis sections $\left\{e_{a}\right\}_{a=1, \cdots, n}$, one has

$$
\Delta^{H} u=-\sum_{a=1}^{n} H_{u}\left(e_{a}, \hat{e}_{a}\right), \text { with } \hat{e}_{a}:=\mathbf{h}\left(e_{a}\right) .
$$

Proof. By definition of horizontal Laplacian.

\section{Finslerian Ricci Deformation and Conformal Metrics}

In this section we prove the main results.

Lemma 5. Let $(M, F)$ be an n-dimensional Finsler manifold. If $\tilde{F}=e^{u} F$ is Finsler metric conformal to $F$ then the horizontal Ricci tensors $\widetilde{\operatorname{Ric}}_{\tilde{F}}^{H}$ and $\mathbf{R i c}_{F}^{H}$ associated with $\tilde{F}$ and $F$ respectively are conformally related by the equation:

$$
\begin{aligned}
\widetilde{\operatorname{Ric}}_{\tilde{F}}^{H}(\xi, X) \\
=\left[\mathbf{R i c}_{F}^{H}+\left(\Delta^{H} u-(n-2)\|\nabla u\|_{g}^{2}\right) g-(n-2)\left(H_{u}-d u \circ d u\right)\right](\xi, X) \\
+(2-n) g(\nabla u, \Theta(X, \mathbf{h}(\xi)))-g\left(\Theta(\mathbf{h}(\nabla u), \mathbf{h}(\xi)), \pi_{*} X\right) \\
-g(\Theta(X, \mathbf{h}(\nabla u)), \xi)+\sum_{a=1}^{n}\left[g\left(\Theta\left(\hat{e}_{a}, \mathbf{h}(\nabla u)\right), e_{a}\right) g\left(\pi_{*} X, \xi\right)\right] \\
+\sum_{a=1}^{n}\left[g\left(\Theta\left(X, \mathbf{h}\left(\Theta\left(\hat{e}_{a}, \mathbf{h}(\xi)\right)\right)\right), e_{a}\right)-g\left(\Theta\left(\hat{e}_{a}, \mathbf{h}(\Theta(X, \mathbf{h}(\xi)))\right), e_{a}\right)\right]
\end{aligned}
$$




$$
+\sum_{a=1}^{n}\left[g\left(\left(\nabla_{X} \Theta\right)\left(\hat{e}_{a}, \mathbf{h}(\xi)\right), e_{a}\right)-g\left(\left(\nabla_{\hat{e}_{a}} \Theta\right)(X, \mathbf{h}(\xi)), e_{a}\right)\right]
$$

where $\left\{e_{a}\right\}_{a=1, \cdots, n}$ is the special $g$-orthonormal basis sections for $\pi^{*} T M$ and $\Theta$ is the $(0,2 ; 1)$-tensor on $(M, F)$ given by

$$
\begin{aligned}
\Theta(X, Y)= & \left(\mathcal{A}\left(\mathcal{B}(X), \pi_{*} Y, \bullet\right)\right)^{\#}+\left(\mathcal{A}\left(\mathcal{B}(Y), \pi_{*} X, \bullet\right)\right)^{\#} \\
& -\left(\mathcal{A}\left(\pi_{*} X, \pi_{*} Y, \mathcal{B}(\mathbf{h}(\bullet))\right)\right)^{\#}
\end{aligned}
$$

with the dual section ( $)^{\#}$ of $\pi^{*} T M$ to the Cartan tensor and the $(0,1 ; 1)$-tensor

$$
\mathcal{B}:=-\frac{1}{F} \frac{\partial}{\partial y^{j}}\left(\frac{F^{2}}{2} g^{i r}-y^{i} y^{r}\right) \frac{\partial u}{\partial x^{r}} \frac{\partial}{\partial x^{i}} \otimes d x^{j} .
$$

Proof. The proof is straightforward from the definitions (0.5) and using the conformal change of the Chern connection given by Theorem 3 .

Lemma 6. Let $(M, F)$ be an $n$-dimensional Finsler manifold. If $\tilde{F}=e^{u} F$ is Finsler metric conformal to $F$ then the horizontal scalar curvatures $\widetilde{\text { Scal }}_{\tilde{F}}^{H}$ and Scal $_{F}^{H}$ associated with $\tilde{F}$ and $F$ respectively are conformally related by the equation:

$$
\begin{aligned}
\widetilde{\mathbf{S c a l}}_{\tilde{F}}^{H}= & e^{-2 u}\left[\mathbf{S c a l}_{F}^{H}+2(n-1) \Delta^{H} u-(n-1)(n-2)\|\nabla u\|_{g}^{2}\right] \\
& +e^{-2 u}\left\{\sum_{b=1}^{n}(2-n)\left[g\left(\nabla u, \Theta_{a b}\right)-g\left(\Theta\left(\hat{e}_{b}, \mathbf{h}(\nabla u)\right), e_{b}\right)\right]\right\} \\
& +e^{-2 u}\left\{\sum_{a, b=1}^{n}\left[g\left(\Theta\left(\hat{e}_{b}, \mathbf{h}\left(\Theta_{a b}\right)\right), e_{a}\right)-g\left(\Theta\left(\hat{e}_{a}, \mathbf{h}\left(\Theta_{a b}\right)\right), e_{a}\right)\right]\right\} \\
& +e^{-2 u}\left\{\sum_{a, b=1}^{n}\left[g\left(\left(\nabla_{\hat{e}_{b}} \Theta\right)_{a b}, e_{a}\right)-g\left(\left(\nabla_{\hat{e}_{a}} \Theta\right)\left(\hat{e}_{b}, \hat{e}_{b}\right), e_{a}\right)\right]\right\}
\end{aligned}
$$

where $\Theta_{a b}:=\Theta\left(\hat{e}_{a}, \hat{e}_{b}\right)$.

Proof. The proof is straightforward from the definitions (0.5) and using the conformal change of the Chern connection given by Theorem 3 .

\subsection{Finslerian Ricci Flow}

One of the advantages of the Finslerian Ricci tensor obtained by contraction of the Chern $h h$-curvature is its relation with the second covariant derivative and hence the horizontal Laplacian operators.

Let $\left(M, F_{t}\right)$ be an $n$-dimensional Finsler manifold of scalar flag curvature and $\left\{g_{t}\right\}_{t \in[0, \tau)}$ the fundamental tensors family of $\left\{F_{t}\right\}_{t \in[0, \tau)}$. We consider the following Finslerian Ricci deformation

$$
\frac{\partial}{\partial t} g_{t}=-2 \mathbf{R i c}_{F_{t}}^{H},\left.\quad g_{t}\right|_{0}=g, \quad \forall t \in[0, \tau),
$$

where $\mathbf{R i c}_{F_{t}}^{H}$ is the horizontal Ricci tensor defined by (19). The existence of solutions is known in special cases, particulary in Riemannian and Berwald spaces, [3]. 


\subsection{Main Results}

We first prove the Theorem 1 on the necessary condition for $F_{t}$ to be conformal equivalent to $F$. We find the existence of a family $\left\{u_{t}\right\}_{t \in[0, \tau)}$ of $C^{\infty}$ functions on $M$ satisfying a parabolic type Equation (2) with initial function equal to zero.

Proof of Theorem 1.1. We denote by $g_{t}$ and $g$ the fundamental tensors of $F_{t}$ and $F$ respectively. If $\tilde{F}_{t} \in\left[F_{t}\right]=[F]$ then there exists a $C^{\infty}$ function $u_{t}$ on $M$ such that $\tilde{F}_{t}=e^{u_{t}} F$ or equivalently $\tilde{g}_{t}=e^{2 u_{t}} g$. Then by definition of Finslerian Ricci flow given by relation (1), we have

$$
\frac{\partial}{\partial t} \tilde{g}_{t}=2 e^{2 u_{t}} g \frac{\partial u_{t}}{\partial t} \text {. }
$$

By Lemma 31 and using the Equation (36), we obtain

$$
\begin{aligned}
& {\left[e^{2 u_{t}}\left(\frac{\partial u_{t}}{\partial t}\right) \underline{g}\right](\xi, X)} \\
& =-\left[\mathbf{R i c}_{F}^{H}+\left(\Delta^{H} u_{t}-(n-2) \|\left.\nabla u_{t}\right|_{g} ^{2}\right) g-(n-2)\left(H_{u_{t}}-d u_{t} \circ d u_{t}\right)\right](\xi, X) \\
& \quad-(2-n) g\left(\nabla u_{t}, \Theta(X, \mathbf{h}(\xi))\right)+g\left(\Theta\left(\mathbf{h}\left(\nabla u_{t}\right), \mathbf{h}(\xi)\right), \pi_{*} X\right) \\
& \quad+g\left(\Theta\left(\mathbf{h}\left(\nabla u_{t}\right)\right), \xi\right)-\sum_{a=1}^{n}\left[g\left(\Theta\left(\hat{e}_{a}, \mathbf{h}\left(\nabla u_{t}\right)\right), e_{a}\right) g\left(\pi_{*} X, \xi\right)\right] \\
& \quad-\sum_{a=1}^{n}\left[g\left(\Theta\left(X, \mathbf{h}\left(\Theta\left(\hat{e}_{a}, \mathbf{h}(\xi)\right)\right)\right), e_{a}\right)-g\left(\Theta\left(\hat{e}_{a}, \mathbf{h}(\Theta(X, \mathbf{h}(\xi)))\right), e_{a}\right)\right] \\
& \quad-\sum_{a=1}^{n}\left[g\left(\left(\nabla_{X} \Theta\right)\left(\hat{e}_{a}, \mathbf{h}(\xi)\right), e_{a}\right)-g\left(\left(\nabla_{\hat{e}_{a}} \Theta\right)(X, \mathbf{h}(\xi)), e_{a}\right)\right]
\end{aligned}
$$

for every $\xi \in \Gamma\left(\pi^{*} T M\right)$ and for every $X \in \Gamma(T \stackrel{\circ}{T} M)$ where $\left\{e_{a}\right\}_{a=1, \cdots, n}$ is the special $g$-orthonormal basis sections for $\pi^{*} T M$. We have

$$
\begin{aligned}
& {\left[e^{2 u_{t}}\left(\frac{\partial u_{t}}{\partial t}\right) g\right]\left(e_{b}, \pi_{*} \hat{e}_{b}\right)} \\
& =-\left[\operatorname{Ric}_{F}^{H}+\left(\Delta^{H} u_{t}-(n-2)\left\|\nabla u_{t}\right\|_{g}^{2}\right) g-(n-2)\left(H_{u_{t}}-d u_{t} \circ d u_{t}\right)\right]\left(e_{b}, \hat{e}_{b}\right) \\
& -(2-n) g\left(\nabla u_{t}, \Theta_{b b}\right)+g\left(\Theta\left(\mathbf{h}\left(\nabla u_{t}\right), \hat{e}_{b}\right), \pi_{*} \hat{e}_{b}\right)+g\left(\Theta\left(\hat{e}_{b}, \mathbf{h}\left(\nabla u_{t}\right)\right), e_{b}\right) \\
& \quad-\sum_{a=1}^{n}\left[g\left(\Theta\left(\hat{e}_{a}, \mathbf{h}\left(\nabla u_{t}\right)\right), e_{a}\right) g\left(\pi_{*} \hat{e}_{b}, e_{b}\right)\right]-\sum_{a=1}^{n} g\left(\Theta\left(\hat{e}_{b}, \mathbf{h}\left(\Theta_{a b}\right)\right), e_{a}\right) \\
& +\sum_{a=1}^{n} g\left(\Theta\left(\hat{e}_{a}, \mathbf{h}\left(\Theta_{b b}\right)\right), e_{a}\right)-\sum_{a=1}^{n}\left[g\left(\left(\nabla_{\hat{e}_{b}} \Theta\right)_{a b}, e_{a}\right)-g\left(\left(\nabla_{\hat{e}_{a}} \Theta\right)_{b b}, e_{a}\right)\right]
\end{aligned}
$$

where $\Theta_{a b}=\Theta\left(\hat{e}_{a}, \hat{e}_{b}\right)$. We then have

$$
\begin{aligned}
\frac{\partial u_{t}}{\partial t}= & -\frac{1}{n} e^{-2 u_{t}} \mathbf{S c a l}_{F}^{H}+\left(\Delta^{H} u_{t}-(n-2)\left\|\nabla u_{t}\right\|_{g}^{2}\right)-\frac{n-2}{n} e^{-2 u_{t}}\left(\Delta^{H} u_{t}-\left\|\nabla u_{t}\right\|^{2}\right) \\
& -\frac{2-n}{n} e^{-2 u_{t}} \sum_{b=1}^{n}\left[g\left(\nabla u_{t}, \Theta_{b b}\right)-g\left(\Theta\left(\mathbf{h}\left(\nabla u_{t}\right), \hat{e}_{b}\right), e_{b}\right)\right]-\frac{1}{n} K_{\Theta}
\end{aligned}
$$

with 


$$
\begin{aligned}
K_{\Theta}= & \sum_{a, b=1}^{n}\left[g\left(\Theta\left(\hat{e}_{b}, \mathbf{h}\left(\Theta\left(\hat{e}_{a}, \hat{e}_{b}\right)\right)\right), e_{a}\right)-g\left(\Theta\left(\hat{e}_{a}, \mathbf{h}\left(\Theta_{b b}\right)\right), e_{a}\right)\right] \\
& +\sum_{a, b=1}^{n}\left[g\left(\left(\nabla_{\hat{e}_{b}} \Theta\right)\left(\hat{e}_{a}, \hat{e}_{b}\right), e_{a}\right)-g\left(\left(\nabla_{\hat{e}_{a}} \Theta\right)\left(\hat{e}_{b}, \hat{e}_{b}\right), e_{a}\right)\right] .
\end{aligned}
$$

From the Equation (39) we obtain the result.

The following Proposition refers to the existence and uniqueness of solution of the Equation (2). We have

Proposition 7. ([8]) Let $\mathcal{E}$ be a bundle of tensors over a smooth compact Riemannian manifold $(M, g)$. We seek a smooth family $[0, \tau) \rightarrow f_{t}$ of smooth tensor fields on $M\left(f_{t} \in C^{\infty}(\mathcal{E})\right)$ which satisfies the equation

$$
\frac{\partial f}{\partial t}=a^{i j}(t, x, f, \nabla f) \nabla_{i} \nabla_{j} f+\psi(t, x, f, \nabla f) \text { with } f(t, x)_{t=0}=\phi(x)
$$

$\phi(x)$ is given and belongs to $C^{\infty}(\mathcal{E})$, the components $a^{i j}$ of a double contravariant symmetric tensor field on $M$ are, in local chart, smooth functions in its arguments, and $\psi$, with values in $\mathcal{E}$, is smooth in its components. If the tensor field $a^{i j}(0, x, f, \nabla f)$ is everywhere positive definite, then there exists a unique smooth solution $f$ on $[0, \tau)$ for some $s \leq T$.

Fom the Proposition 7, we get

Corollary 2. Let $(M, F)$ be an n-dimensional compact Finsler manifold. Assume $n \geq 2$. Then the Equation (2) in Theorem 1 has a unique solution on a parameterized-interval $[0, s)$ for some $s \leq T$.

Proof. We put: $\mathcal{E}=C^{\infty}(M)$,

$$
a^{i j}(t, x, f, \nabla f) \nabla_{i} \nabla_{j} f(t)=\frac{2(n-1)}{n} e^{-2 u_{t}} \delta^{a b} \nabla_{a} \nabla_{b} u_{t}
$$

and

$$
\begin{aligned}
\psi & (t, x, f, \nabla f) \\
= & -\frac{1}{n} e^{-2 u_{t}}\left[\mathbf{S c a l}_{F}^{H}-(n-1)(n-2)\left\|\nabla u_{t}\right\|_{g}^{2}\right] \\
& -\frac{2-n}{n} e^{-2 u_{t}} \sum_{b=1}^{n}\left[g\left(\nabla u_{t}, \Theta_{a b}\right)-g\left(\Theta\left(\hat{e}_{b}, \mathbf{h}\left(\nabla u_{t}\right)\right), e_{b}\right)\right] \\
& -\frac{1}{n} e^{-2 u_{t}} \sum_{a, b=1}^{n}\left[g\left(\Theta\left(\hat{e}_{b}, \mathbf{h}\left(\Theta\left(\hat{e}_{a}, \hat{e}_{b}\right)\right)\right), e_{a}\right)-g\left(\Theta\left(\hat{e}_{a}, \mathbf{h}\left(\Theta_{a b}\right)\right), e_{a}\right)\right] \\
& -\frac{1}{n} e^{-2 u_{t}} \sum_{a, b=1}^{n}\left[g\left(\left(\nabla_{\hat{e}_{b}} \Theta\right)\left(\hat{e}_{a}, \hat{e}_{b}\right), e_{a}\right)-g\left(\left(\nabla_{\hat{e}_{a}} \Theta\right)\left(\hat{e}_{b}, \hat{e}_{b}\right), e_{a}\right)\right] .
\end{aligned}
$$

Then the relations (41) and (2) are equivalent with $\left.u_{t}\right|_{t=0}=0$.

Moreover,

$$
a^{i j}(0, x, f, \nabla f)=\frac{2(n-1)}{n} \delta^{a b}
$$

which is positive definite since $\frac{2(n-1)}{n}$ is positive and $\delta^{a b}$ is nonnegative. Applying the Proposition 7 we obtain the result. 
When a Finsler metric $F$ is of Einstein type, we obtain the existence on a compact Finsler-Einstein manifold of a Ricci flow which preserves the conformal class.

Proof of Theorem 1.2. For $t \in[0, s), \quad \tilde{g}(t):=e^{2 u_{t}} g$. Since $\left.u_{t}\right|_{t=0}=0$, we have

$$
\left.\tilde{g}_{t}\right|_{t=0}=e^{2 u_{0}} g=g \text {. }
$$

It follows that, since $u_{t}$ is solution of Equation (2),

$$
\begin{aligned}
\frac{\partial}{\partial t} \tilde{g}(t)= & 2 e^{2 u_{t}} g \frac{\partial u_{t}}{\partial t}=-2\left\{\frac{1}{n} \mathbf{S c a l}_{F}^{H} \pi^{*} g+\left(\Delta^{H} u_{t}-(n-2)\left\|\nabla u_{t}\right\|_{g}^{2}\right) g\right. \\
& +\frac{n-2}{n}\left(\Delta^{H} u_{t}+\left\|\nabla u_{t}\right\|_{g}^{2}\right) g+\frac{2-n}{n} \sum_{b=1}^{n} g\left(\nabla u_{t}, \Theta_{a b}\right) g \\
& +\frac{2}{n} \sum_{b=1}^{n} g\left(\Theta\left(\hat{e}_{b}, \mathbf{h}\left(\nabla u_{t}\right)\right), e_{b}\right) g-\sum_{b=1}^{n} g\left(\Theta\left(\hat{e}_{b}, \mathbf{h}\left(\nabla u_{t}\right)\right), e_{b}\right) g \\
& +\frac{1}{n} \sum_{a, b=1}^{n}\left[g\left(\Theta\left(\hat{e}_{b}, \mathbf{h}\left(\Theta\left(\hat{e}_{a}, \hat{e}_{b}\right)\right)\right), e_{a}\right)-g\left(\Theta\left(\hat{e}_{a}, \mathbf{h}\left(\Theta_{a b}\right)\right), e_{a}\right)\right] g \\
& \left.+\frac{1}{n} \sum_{a, b=1}^{n}\left[g\left(\left(\nabla_{\hat{e}_{b}} \Theta\right)\left(\hat{e}_{a}, \hat{e}_{b}\right), e_{a}\right)-g\left(\left(\nabla_{\hat{e}_{a}} \Theta\right)\left(\hat{e}_{b}, \hat{e}_{b}\right), e_{a}\right)\right] g\right\} .
\end{aligned}
$$

If $F$ is a Finsler-Einstein metric, the trace-free horizontal Ricci tensor associated to $F$ vanishes and then have

$$
\mathbf{R i c}_{F}^{H}=\frac{1}{n}\left(\operatorname{Scal}_{F}^{H} \pi^{*} g\right)
$$

If the tensor $T_{t}$ is conformally equivalent to $g$, then it holds

$$
\begin{aligned}
& T_{c d}(t)=\frac{\operatorname{trace} T_{t}}{n} \delta c d \\
& =\frac{n-2}{n}\left(\Delta^{H} u_{t}+\left\|\nabla u_{t}\right\|_{g}^{2}\right) e^{2 u_{t}} \delta c d+\frac{2-n}{n} \sum_{b=1}^{n} g\left(\nabla u_{t}, \Theta_{a b}\right) e^{2 u_{t}} \delta c d \\
& +\frac{2}{n} \sum_{b=1}^{n} g\left(\Theta\left(\hat{e}_{b}, \mathbf{h}\left(\nabla u_{t}\right)\right), e_{b}\right) e^{2 u_{t}} \delta c d-\sum_{a=1}^{n} g\left(\Theta\left(\hat{e}_{b}, \mathbf{h}\left(\nabla u_{t}\right)\right), e_{b}\right) e^{2 u_{t}} \delta c d \\
& +\frac{1}{n} \sum_{a, b=1}^{n} g\left(\Theta\left(\hat{e}_{b}, \mathbf{h}\left(\Theta\left(\hat{e}_{a}, \hat{e}_{b}\right)\right)\right), e_{a}\right) e^{2 u_{t}} \delta c d-\frac{1}{n} \sum_{a, b=1}^{n} g\left(\Theta\left(\hat{e}_{a}, \mathbf{h}\left(\Theta_{a b}\right)\right), e_{a}\right) e^{2 u_{t}} \delta c d \\
& +\frac{1}{n} \sum_{a, b=1}^{n} g\left(\left(\nabla_{\hat{e}_{b}} \Theta\right)\left(\hat{e}_{a}, \hat{e}_{b}\right), e_{a}\right) e^{2 u_{t}} \delta c d-\frac{1}{n} \sum_{a, b=1}^{n} g\left(\left(\nabla_{\hat{e}_{a}} \Theta\right)\left(\hat{e}_{b}, \hat{e}_{b}\right), e_{a}\right) e^{2 u_{t}} \delta c d .
\end{aligned}
$$

Putting (43) and (44) in (42), we obtain

$$
\begin{aligned}
& {\left[\frac{\partial}{\partial t} \tilde{g}(t)\right]\left(e_{b}, \pi_{*} \hat{e}_{b}\right)=-2\left\{\mathbf{R i c}_{F}^{H}+\left(\Delta^{H} u_{t}-(n-2)\left\|\nabla u_{t}\right\|_{g}^{2}\right) \underline{g}+T_{t}\right\}\left(e_{b}, \pi_{*} \hat{e}_{b}\right) } \\
= & -2\left[\mathbf{R i c}_{F}^{H}+\left(\Delta^{H} u-(n-2)\|\nabla u\|_{g}^{2}\right) \underline{g}-(n-2)\left(H_{u}-d u \circ d u\right)\right]\left(e_{b}, \hat{e}_{b}\right) \\
& -(2-n) g\left(\nabla u, \Theta_{b b}\right)+g\left(\Theta\left(\mathbf{h}(\nabla u), \hat{e}_{b}\right), \pi_{*} \hat{e}_{b}\right)+g\left(\Theta\left(\hat{e}_{b}, \mathbf{h}(\nabla u)\right), e_{b}\right) \\
& -\sum_{a=1}^{n}\left[g\left(\Theta\left(\hat{e}_{a}, \mathbf{h}(\nabla u)\right), e_{a}\right) g\left(\pi_{*} \hat{e}_{b}, e_{b}\right)\right] \\
& -\sum_{a=1}^{n}\left[g\left(\Theta\left(\hat{e}_{b}, \mathbf{h}\left(\Theta_{a b}\right)\right), e_{a}\right)-g\left(\Theta\left(\hat{e}_{a}, \mathbf{h}\left(\Theta_{b b}\right)\right), e_{a}\right)\right]
\end{aligned}
$$




$$
-\sum_{a=1}^{n}\left[g\left(\left(\nabla_{\hat{e}_{b}} \Theta\right)\left(\hat{e}_{a}, \hat{e}_{b}\right), e_{a}\right)-g\left(\left(\nabla_{\hat{e}_{a}} \Theta\right)\left(\hat{e}_{b}, \hat{e}_{b}\right), e_{a}\right)\right]=-2 \widetilde{\operatorname{Ric}}_{\tilde{F}}^{H} .
$$

Hence, $\{\tilde{F}(t)\}_{t \in[0, s)}$ is a Ricci deformation of $F$, by construction of $\left[g_{t}\right]=[g]$, $\forall t \in[0, s)$. The uniqueness derives from Theorem 1 and Corollary 2 .

Remark 3.1. If $F$ is a Riemannian metric then, by the relation (32), the $(0,2,1)$-tensor $\Theta$ vanishes. The Theorem 2 becomes the result in [9] for the Riemannian case.

\section{Conclusions}

In this present work, we use the pulled-back bundle approach [2] to introduce a Finslerian horizontal Ricci flow, called Finslerian Ricci deformation. This approach is natural and is very important because it facilitates the analogy with the Riemannian geometry.

Using the results of the previous sections we plan to study, in the future, the evolution of the Chern $h h$-curvature and its various traces under the Finslerian Ricci deformation.

\section{Conflicts of Interest}

The authors declare no conflicts of interest regarding the publication of this paper.

\section{References}

[1] Bidabad, B., Shahi, A. and Ahmadi, E.Y. (2017) Deformation of Cartan Curvature on Finsler Manifolds. Bulletin of the Korean Mathematical Society, 54, 2119-2139.

[2] Bao, D., Chern, S.S. and Shen, Z. (2000) An Introduction to Riemannian-Finsler Geometry. Springer-Verlang, New York, 1-67. https://doi.org/10.1007/978-1-4612-1268-3

[3] Azami, S. and Razavi, A. (2013) Existence and Uniqueness for Solution of Ricci Flow on Finsler Manifolds. International Journal of Geometric Methods in Modern Physics, 10, 1-21. https://doi.org/10.1142/S0219887812500910

[4] Djelid, R. (2011) Déformations conformes des variétés de Finsler-Ehresmann. Thèse numéro 5032 EPFL, Faculté des Sciences, Ecole Polytechnique Fédérale de Lausanne, 1-103.

[5] Shen, Z. (1997) Volume Comparison and Its Applications in Riemann-Finsler Geometry. Advances in Mathematics, 128, 306-328.

https://doi.org/10.1006/aima.1997.1630

[6] Mbatakou, J.S. and Todjihounde, L. (2014) Conformal Change of Finsler-Ehresmann Connections. Applied Sciences, Balkan Society of Geometers, 16, 32-47.

[7] Chern, S.S. and Shen, Z. (2005) Riemann-Finsler Geometry. Nankai Tracts in Mathematics by Word Scientific Publishing, 6, 51-63. https://doi.org/10.1142/9789812567864_0003

[8] Aubin, T. (1998) Some Nonlinear Problems in Riemannian Geometry. Springer-Verlag, Berlin Heidelberg, 323-325. https://doi.org/10.1007/978-3-662-13006-3 
G. Nibaruta et al.

[9] Todjihounde, L. (2003) Ricci Deformation and Conformal Change of Metrics. Bulletin of the Calcutta Mathematical Society, 95, 361-366. 